Ciência Florestal, Santa Maria, v. 21, n. 2, p. 335-347, abr.-jun., 2011

ISSN 0103-9954

\title{
CARACTERIZAÇÃO DE COMPÓSITOS PRODUZIDOS COM POLIETILENO DE ALTA DENSIDADE (HDPE) E SERRAGEM DA INDÚSTRIA MOVELEIRA - PARTE II - EXTRUSÃO EM DUPLA-ROSCA ${ }^{1}$
}

\author{
CHARACTERIZATION OF COMPOSITES MADE OF HDPE AND FURNITURE INDUSTRY \\ SAWDUST. PART II: DOUBLE-SCREW EXTRUSION \\ Éverton Hillig ${ }^{2}$ Setsuo Iwakiri ${ }^{3}$ Clovis Roberto Haselein ${ }^{4}$ \\ Otávio Bianchi ${ }^{5}$ Débora Moraes Hillig ${ }^{6}$
}

\begin{abstract}
RESUMO
Neste trabalho, realizou-se a caracterização de compósitos confeccionados com polietileno virgem de alta densidade (v-HDPE) e diferentes tipos de serragem gerados na indústria moveleira. O equipamento usado foi uma extrusora de dupla-rosca corrotante, de $19 \mathrm{~mm}$, complementando trabalho anterior no qual os compósitos foram misturados numa extrusora mono-rosca. Utilizou-se temperatura de $180^{\circ} \mathrm{C}$ nas cinco zonas de aquecimento, rotação de $150 \mathrm{rpm}$ e vazão de $1 \mathrm{~kg} \cdot \mathrm{h}^{-1}$. Foram utilizados resíduos de madeira de pinus (Pinus taeda), de chapa de fibra de média densidade (MDF) e madeira de eucalipto (Eucalyptus grandis), que foram incorporados ao HDPE "virgem" juntamente com um agente de acoplamento a base de anidrido maleico. Para caracterização física dos compósitos foram realizadas análises por calorimetria diferencial de varredura (DSC) e por microscopia eletrônica de varredura (SEM). Foram também analisadas as propriedades mecânicas de resistência à tração, flexão estática e resistência ao impacto, conforme as normas ASTM D638, D790 e D256 respectivamente. Verificou-se que todos os tipos de serragem agiram como agentes nucleantes, pois os compósitos apresentaram índices de cristalinidade maiores que o v-HDPE puro. Também se observou que houve influência do tipo de serragem usada na dispersão das fibras na matriz polimérica. Os ensaios mecânicos mostraram diferenças nas propriedades dos compósitos confeccionados com diferentes tipos de serragem. De maneira geral, inclusão de serragem de MDF proporcionou compósitos com maior resistência à flexão e ao impacto que aqueles fabricados com eucalipto e pinus. Comparando os valores obtidos nesse trabalho com os obtidos na extrusão com monorrosca, conclui-se que foi possível transferir as propriedades da madeira para o compósito de melhor forma na extrusão com dupla-rosca.
\end{abstract}

Palavras-chave: compósitos; HDPE; serragem.

\begin{abstract}
In this work, wood plastic composites made of HDPE and different types of wood sawdust generate at furniture industries are characterised. The equipment used was a $19 \mathrm{~mm}$ co-rotating twin-screw extruder, complementing previous studies where the composites were mixed using a single-screw extruder. Temperatures of $180^{\circ} \mathrm{C}$ were applied at the five heating zones, rotating at $150 \mathrm{rpm}$ with a flux of $1 \mathrm{~kg} \cdot \mathrm{h}^{-1}$. Residues of MDF, of loblolly pine and of eucalypt wood were used, and were mixed into the HDPE with a coupling agent (anhydride maleic). Physical characterization of the composites was performed by differential scanning calorimetry (DSC) and by scanning electronic microscopy. In addition, the mechanical

1. Parte da Tese de Doutorado do $1^{\circ}$ autor

2. Engenheiro Florestal, Dr., Professor Adjunto do Departamento de Engenharia Florestal, Universidade Estadual do Centro-Oeste, PR 153, Km 07, Riozinho, CEP 84500-000, Irati (PR). ehillig@irati.unicentro.br

3. Engenheiro Florestal, Dr., Professor Titular do Departamento de Engenharia e Tecnologia Florestal, Setor de Ciências Agrárias, Universidade Federal do Paraná, CEP 80210-170, Curitiba (PR). setsuo@floresta.ufpr.br

4. Engenheiro Florestal, PhD., Professor Associado do Departamento de Ciências Florestais da Universidade Federal de Santa Maria, Av. Roraima, 1000, CEP 97015-900, Santa Maria (RS). clovis.haselein@smail.ufsm.br

5. Engenheiro Químico, Doutorando do Programa de Pós-Graduação em Ciência dos Materiais, Universidade Federal do Rio Grande do Sul, Porto Alegre (RS). otavio.bianchi@ufrgs,br

6. Acadêmica do curso de Engenharia Elétrica, Universidade de Caxias do Sul, Alameda João Dal Sasso, 800, CEP 95700-000, Bento Gonçalves (RS).dmhillig@ucs.br

Recebido para publicação em 9/04/2009 e aceito em 10/09/2010
\end{abstract}

Ci. Fl., v. 21, n. 2, abr.-jun., 2011 
properties of tension, static bending and impact were analysed, according to ASTM D638, D790 and D256. All types of sawdust acted as a nucleate agent, since the composites showed a crystallinity index higher than pure HDPE. Also, it was observed that the type of sawdust influenced fibre's dispersion in the polymeric matrix. The mechanical tests showed differences in the properties of the composites made of different types of sawdust. In general, inclusion of MDF sawdust resulted in composites with higher bending strength and impact work than those containing eucalypt and pine. Comparing results obtained using a single-screw extruder and values obtained in this study, it can be concluded that the properties of the wood are more effectively transferred to the composite using a double-screw extruder.

Keywords: composites; HDPE; sawdust.

\section{INTRODUÇÃO}

No Brasil, ainda existem poucas aplicações comerciais de compósitos polímeromadeira. Existem algumas iniciativas isoladas de fabricação desses compósitos, desenvolvidas por pequenas empresas que, em geral, procuram adaptar a tecnologia existente para processamento de termoplásticos na fabricação de uma matériaprima destinada a empresas que fabricam produtos por injeção. Também, existem iniciativas na fabricação de um produto final que pode ser processado por extrusão, injeção, compressão ou, ainda, uma combinação de dois desses processos de transformação.

Ruch et al. (2004) citam os principais processos para a preparação e moldagem de componentes confeccionados com materiais compósitos de matriz termoplástica reforçados com fibras naturais, que são: extrusão, injeção, plastificação/prensagem, preparação de formulação/injeção e processo NMT (naturfasermattenverstärkter) que é utilizado para termoplásticos reforçados com mantas de fibras naturais. Num trabalho para desenvolvimento de um processo para a preparação e moldagem de compósitos de polipropileno com fibras naturais, os autores verificaram ser possível a transformação direta dos materiais de partida num produto final, por meio de uma máquina para preparação de formulação e injeção, fabricada em Munique, na Alemanha.

O mais comum, entretanto, são os processos de mistura e de conformação dos compósitos poliméricos reforçados com fibras celulósicas serem realizados em duas etapas. Numa primeira etapa, faz-se a incorporação das fibras à matriz, seguido de um processamento de moldagem do produto final. Diversos tipos de processos podem ser empregados em ambas as fases, mas existe uma tendência, tanto na indústria como no desenvolvimento de trabalhos científicos, de se utilizar a extrusão dos materiais em extrusora de dupla-rosca, seguido da injeção da mistura para obtenção do produto final (Saheb e Jog, 1999).

É importante ressaltar que o uso de diferentes métodos de processamento pode influenciar as propriedades físico-mecânicas dos compósitos. Stark et al. (2003) afirmaram que diferentes métodos de produção de compósitos polímeromadeira, ocasionam diferentes características na face do material produzido. Num estudo do efeito do método de processamento no envelhecimento acelerado de compósitos de HDPE e serragem de madeira, os autores produziram amostras dos compósitos por injeção, por extrusão e por extrusão seguida de aplainamento do material. Observaram que os diferentes métodos de processamento produziram faces com diferentes componentes.

Bledzki et al. (2005) estudaram o comportamento de compósitos confeccionados com PP e madeiras de coníferas e folhosas, submetidos a três diferentes formas de processamento das misturas. Misturador de rolos, misturador termocinético e extrusora de dupla-rosca foram usados para homogeneizar as misturas de PP com madeira. Foram também testados três tipos de agentes compatibilizantes. Foi verificado que dos três processos empregados, a extrusão proporcionou melhores propriedades mecânicas e valores mais baixos de absorção d'água nos compósitos. Uma análise por microscopia revelou danos aparentes nas fibras submetidas ao processamento por misturador de rolos e por misturador temocinético.

Clemons e Ibach (2004) estudaram os efeitos do tipo de processamento de compósitos de HDPE reciclado reforçado com serragem de pinus, na resistência ao ataque de fungos e absorção de umidade. Os autores verificaram que os compósitos confeccionados por injeção foram mais resistentes em todos os aspectos que os confeccionados por compressão. Estes, por sua vez, foram mais resistentes que os extrusados.

De acordo com Billmeyer Jr. (1971), no 
processo de extrusão, o polímero é propelido continuamente ao longo de uma rosca com regiões de alta temperatura e pressão onde é fundido e compactado, sendo finalmente forçado por uma ferramenta para se obter um produto final. Para se obter a temperatura de fusão do polímero, algum calor externo é aplicado, mas grande parte do calor é gerada por fricção. Máquinas extrusoras modernas podem ser providas de dupla-rosca contra-rotante, em que duas roscas giram lado a lado em direções opostas promovendo maior trabalho na massa fundida. Noutras, chamadas extrusoras com sistema de degasagem, existem aberturas ou saídas de ar em alguns pontos ao longo da rosca, para extração de voláteis do polímero fundido.

Segundo Rose (2002), os maiores problemas encontrados para extrusão de compósitos polímerosmadeira são as características da serragem, como baixa densidade, fluxo não livre e alta absorção de umidade. Esse fato se agrava quando esses compósitos contêm maior quantidade em peso de serragem do que de resina. Assim, o autor salienta que, para extrusão de compósitos polímero-madeira, o transporte e a mistura dos materiais necessitam de equipamentos diferentes dos utilizados para os plásticos convencionais.

Em TECNOLOGIA (2010), são transcritas as peculiaridades do processo de coextrusão de fibras de madeira e polímeros. Segundo o artigo, extrusoras de roscas duplas (contra-rotativas) podem ser usadas para compósitos com madeira quando sua umidade for inferior a 1\%. Para umidades maiores e visando à melhor homogenização da mistura, devem ser usadas extrusoras projetadas para compósitos de polímeros com madeira, que podem remover a umidade das fibras, mesmo em níveis altos (8\%). Existem duas diferenças básicas dessas extrusoras em relação às convencionais: que o processo inclui uma extrusora principal de dupla-rosca paralela contra-rotativa e outra satélite para o polímero, e que as fibras de madeira são submetidas a uma zona de vazão para remoção da umidade. Com esse tipo de equipamento, segundo o artigo, pode-se conseguir misturas com até $80 \%$ de proporção de serragem.

Por outro lado, tem-se verificado que menores proporções de serragem podem ser incorporadas ao polímero utilizando equipamentos convencionais de processamento de polímeros termoplásticos. Vianna et al (2004), Yamaji (2004), Fonseca (2005), Hillig et al (2008) são alguns exemplos de trabalhos desenvolvidos com equipamentos convencionais, extrusoras mono ou dupla-rosca co-rotante, na qual proporções de até $40 \%$ de serragem de madeira foram incorporadas a polímeros termoplásticos. É importante, assim, que sejam realizados mais estudos das características dos materiais processados com esses equipamentos. Dessa forma, complementando um trabalho de fabricação de compósitos em extrusora mono-rosca, foram produzidos e caracterizados compósitos polímero-madeira numa extrusora dupla-rosca co-rotante, utilizando diferentes tipos e proporçõesde serragem de madeira.

\section{MATERIAIS E MÉTODOS}

\section{Materiais}

Foi utilizado resíduo de madeira de pinus (Pinus taeda), chapa de fibra de média densidade (MDF) e serragem de madeira de eucalipto (Eucalyptus grandis). Os resíduos de pinus e de MDF foram obtidos na forma de serragem, respectivamente das operações de corte com serra de fita e com serra circular. Como não foi possível obter serragem pura de eucalipto, essa serragem foi gerada num moinho de martelo, utilizando cavacos de eucalipto. A madeira de eucalipto foi obtida numa indústria de celulose, com tamanho variando entre 2 a $4 \mathrm{~cm}$ de comprimento e 1 a $2 \mathrm{~cm}$ de largura.

$\mathrm{Na}$ fase matricial dos compósitos foi usado polietileno de alta densidade virgem - v-HDPE (HC 7260 LS), da Ipiranga Petroquímica. As propriedades do v-HDPE são apresentadas na Tabela 1.

TABELA 1: Propriedades do v-HDPE utilizado para confecção dos compósitos.

TABLE 1: v-HDPE properties used for composite manufacturing.

\begin{tabular}{lccc}
\hline Propriedade & Norma & Unidade & Valores \\
\hline Densidade & ASTM D 792 - 98 & $\mathrm{g} / \mathrm{cm}^{3}$ & $0,959 \pm 0,003$ \\
\hline MFI 190/2,16 & ASTM D 1238 - 01 & $\mathrm{g} / 10 \mathrm{~min}$ & $8,0 \pm 1,6$ \\
\hline Tensão no Escoamento (em placa) & ASTM D 638 - 02 & $\mathrm{Mpa}$ & 31 \\
\hline Dureza Shore D/15 & ASTM D 2240 - 03 & - & 65 \\
\hline Resistência ao Impacto IZOD a 23 ${ }^{\circ} \mathrm{C}$ & ASTM D 256 - 03 & $\mathrm{J} / \mathrm{m}$ & 35 \\
\hline Temperatura de Amolecimento Vicat 9,8 N & ASTM D 1525 - 04 & ${ }^{\circ} \mathrm{C}$ & 126 \\
\hline
\end{tabular}

FONTE: Braskem, 2010 
Foi também utilizado o agente de acoplamento de nome comercial Polybond 3009 para melhorar a interação entre as fases de fibra e de matriz. Conforme o fabricante, esse aditivo tratase de um HDPE graftizado com anidrido maleico, recomendado como agente de acoplamento para vidro, mica, Alumina Trihidratada (ATH), fibras de madeira e outras fibras usadas em compósitos com polietileno. Também é recomendado como compatibilizante para blendas de polietileno com polímeros polares como nylon e EVOH. As propriedades físicas que caracterizam esse produto são apresentadas na Tabela 2.

TABELA 2: Propriedades físicas do polybond 3009. TABLE 2: $\quad$ Physical properties of polybond 3009.

\begin{tabular}{lcc}
\hline Propriedades & $\begin{array}{c}\text { Valores } \\
\text { médios }\end{array}$ & $\begin{array}{c}\text { Método de } \\
\text { determinação }\end{array}$ \\
\hline Forma física & Pellets & \\
\hline $\begin{array}{l}\text { Índice de fluidez } \\
(190 / 2.16)\end{array}$ & $5 \mathrm{~g} / 10 \mathrm{~min}$. & $\begin{array}{c}\text { ASTM D } \\
1238\end{array}$ \\
\hline $\begin{array}{l}\text { Densidade }\left(23^{\circ} \mathrm{C}\right) \\
\begin{array}{l}\text { Conteúdo de anidrido } \\
\text { maleico }\end{array}\end{array}$ & $0,95 \mathrm{~g} / \mathrm{cm}^{3}$ & $\begin{array}{c}\text { ASTM D } \\
792\end{array}$ \\
\hline
\end{tabular}

Temperatura de fusão $127^{\circ} \mathrm{C}$ DSC

FONTE: Chemtura, 2010.

\section{Caracterização dos diferentes tipos de serragem utilizadas nos experimentos}

A caracterização dos diferentes tipos de serragem utilizadas foi realizada utilizando-se análise granulométrica, análise termogravimétrica (TGA) e termogravimetria derivativa (DTG).

A caracterização granulométrica das amostras de madeira foi realizada utilizando peneiras da série Tyler (série fina), com peneiras variando de 8 Mesh Tyler até 400 Mesh Tyler. O ensaio foi realizado com auxílio de um agitador mecânico da Produtest durante um período de 20 minutos.

As análises termogravimétricas (TGA e DTG) das amostras de madeira foram realizadas em um equipamento Shimadzu, modelo TGA-50 com taxa de aquecimento de $10{ }^{\circ} \mathrm{C} \cdot \mathrm{min}^{-1}$, sob atmosfera de nitrogênio, com um fluxo de $50 \mathrm{ml} \cdot \mathrm{min}^{-1}$. As condições de ensaio foram da temperatura ambiente até $900^{\circ} \mathrm{C}$, com as massas das amostras variando entre 10 a $15 \mathrm{mg}$. Foram analisadas três amostras de cada tipo de serragem.

\section{Confecção dos compósitos}

A serragem foi incorporada ao HDPE, juntamente com $5 \%$ em peso do agente de acoplamento Polybond 3009, por meio de extrusão com extrusora dupla-rosca, nas proporções de 20, 30 e $40 \%$ para pinus e MDF e de 20 e $30 \%$ para eucalipto, pois não foi possível sua extrusão na proporção de $40 \%$.

A mistura HDPE/serragem/Polybond foi realizada manualmente e a alimentação foi realizada com um dosador gravimétrico. Utilizaram-se uma extrusora Baker \& Perkins com diâmetro de rosca de $19 \mathrm{~mm}$ e razão comprimento/diâmetro (L/D) de 25. Foram usadas temperaturas de $180^{\circ} \mathrm{C}$ nas cinco zonas de aquecimento, rotação de $150 \mathrm{rpm}$ e vazão de $1 \mathrm{~kg} \cdot \mathrm{h}^{-1}$. O material extrusado foi transformado em pellets com picotador, após resfriamento em água na saída da extrusora, e acondicionado em sacos plásticos.

\section{Confecção dos corpos-de-prova}

A confecção dos corpos-de-prova foi realizada por meio de uma ferramenta de injeção, segundo as normas ASTM D638, D790 e D256 para os ensaios de tração, flexão estática e impacto respectivamente. $\mathrm{Na}$ injeção das misturas, em injetora HIMACO-150, foram usados os parâmetros apresentados na Tabela 3. Após a confecção, os corpos-de-prova foram condicionados em sala climatizada, a temperatura de $20^{\circ} \mathrm{C}$ e $50 \%$ de umidade relativa, para realização dos ensaios. De todos os corpos-de-prova, foram tomadas as medidas médias e a massa, de forma a determinar a massa específica aparente de cada um. A massa específica aparente foi determinada pela razão entre a massa e o volume do corpo-de-prova condicionado.

TABELA 3: Parâmetros de injeção dos corpos-de-prova.

TABLE 3: Injection parameters of the samples.

\begin{tabular}{l|l|l|l|c|c}
\hline \multicolumn{1}{c|}{ Parâmetro } & \multicolumn{1}{c}{ Unidade } & \multicolumn{1}{c}{ Valor } & \multicolumn{1}{c}{ Parâmetro } & Unidade & Valor \\
\hline Temperatura do bico & ${ }^{\circ} \mathrm{C}$ & 90 & Força de injeção & ton & 50 \\
\hline Temperatura da zona 1 & ${ }^{\circ} \mathrm{C}$ & 180 & Força de recalque & ton & 20 \\
\hline Temperatura da zona 2 & ${ }^{\circ} \mathrm{C}$ & 170 & Força de dosagem & ton & 90 \\
\hline Temperatura da zona 3 & ${ }^{\circ} \mathrm{C}$ & 160 & Força de descompressão & ton & 70 \\
\hline Tempo de injeção & $\mathrm{S}$ & 5 & Tempo de resfriamento & $\mathrm{s}$ & 15 \\
\hline
\end{tabular}

Ci. Fl., v. 21, n. 2, abr.-jun., 2011 


\section{Caracterização física dos compósitos}

A caracterização física dos compósitos foi realizada por meio de calorimetria diferencial de varredura (DSC). As amostras com massas variando entre 9 e $10 \mathrm{mg}$ foram submetidas ao ensaio calorimétrico em um equipamento DSC-50 da Shimadzu, sob atmosfera de nitrogênio, com um fluxo de $50 \mathrm{ml} \cdot \mathrm{min}^{-1}$. As amostras foram aquecidas da temperatura ambiente até $200^{\circ} \mathrm{C}$, a velocidade de $10^{\circ} \mathrm{C} \cdot \mathrm{min}^{-1}$ e mantidas durante aproximadamente 5 minutos, após foram resfriadas a $10^{\circ} \mathrm{C} \cdot \mathrm{min}^{-1}$ até a temperatura ambiente e mantidas em uma isoterma por um período de 5 minutos. Para o segundo ciclo de aquecimento, as amostras foram submetidas às mesmas condições adotadas no primeiro. Para essa análise, utilizaram-se índio e zinco metálico para a calibração da energia e temperatura.

Foram analisados os índices de cristalinidade e a cristalinidade relativa das misturas dos diferentes tipos de serragem, somente na proporção de $30 \%$ serragem e $70 \%$ HDPE, com objetivo de verificar a influência da serragem nessa proporção. Foram analisadas três amostras de cada compósito e do HDPE puro, sendo os índices de cristalinidade submetidos à ANOVA e teste de Tukey para comparação entre médias.

Para análise da dispersão da serragem na matriz polimérica, realizou-se microscopia eletrônica de varredura (SEM), em microscópio eletrônico marca JEOL, modelo ISM - 5800 do Centro de Microscopia Eletrônica da UFRGS, com ampliações variando entre 200 e 10000 vezes, e $20 \mathrm{KV}$ de energia de feixe incidente. As amostras foram imersas em nitrogênio líquido por 5 minutos e fraturadas por impacto. A superfície fraturada foi recoberta por uma camada de ouro e as amostras foram coladas em suportes individualizados confeccionados com aço inoxidável, no formato de pequenos cilindros com $1,2 \mathrm{~cm}$ de diâmetro por 1 $\mathrm{cm}$ de altura.

\section{Análise dos ensaios físico-mecânicos}

Foi realizado um experimento fatorial incompleto, no delineamento inteiramente casualizado, com dois fatores: tipo de serragem e proporção de serragem. Foram usados dois tipos de serragem (Pinus taedae e MDF) em três proporções $(20 \%, 30 \%$ e $40 \%)$, mais um tipo de serragem (Eucalyptus grandis) em duas proporções (20 e 30\%). Também foi usado apenas HDPE puro, totalizando nove tratamentos com cinco repetições.
A análise dos resultados das propriedades físico-mecânicas foi realizada por meio de análise de variância (ANOVA). Para comparação entre as médias das propriedades nos diferentes tratamentos, quando estabelecidas as diferenças pela ANOVA, foi realizado teste de Tukey, ao nível de 5\% de significância.

\section{RESULTADOS E DISCUSSÃO}

\section{Caracterização das serragens utilizadas nos experimentos (granulometria e TGA)}

A análise granulométrica dos diferentes tipos de serragens utilizados nos experimentos mostra que a distribuição do tamanho das partículas varia de um tipo de serragem para outro. A Figura 1 apresenta a frequência média de cada tamanho de partícula para cada tipo de serragem usada. Verificase que a serragem de MDF é a que apresenta uma distribuição por tamanho mais heterogêneo, em comparação com os outros tipos, apresentando um percentual de frequência significativo em todas as classes de tamanho. Por ordem de heterogeneidade de distribuição de frequência, seguem as serragens de eucalipto e de pinus. Com relação ao diâmetro médio superficial, foi encontrado para o MDF um valor de $72 \mu \mathrm{m}$, para o pinus de $110 \mu \mathrm{m}$ e para eucalipto de $170 \mu \mathrm{m}$.

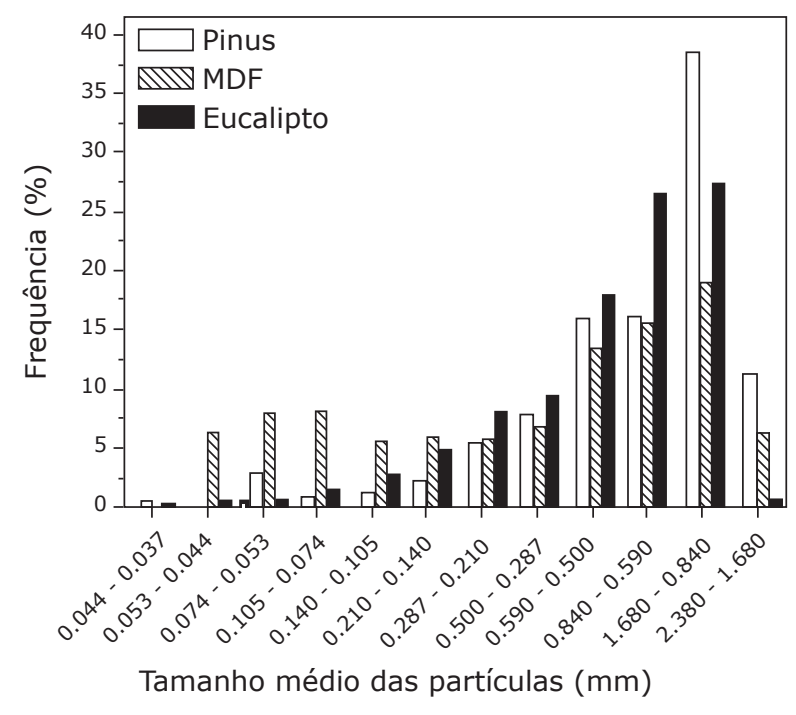

FIGURA 1: Distribuição de freqüência do tamanho médio das partículas, dos diferentes tipos de serragens usadas nos experimentos.

FIGURE 1: Frequency distribution of average size of the particles of the different types of sawdust used in the experiments. 
A Figura 2 apresenta a perda percentual de massa das amostras de serragem das madeiras utilizadas em função da temperatura. Nota-se que as curvas, para cada tipo de serragem, apresentaramse semelhantes, com faixas de temperatura de perda de massa muito próximas. Por outro lado, as perdas de massa de cada tipo de serragem apresentaram diferenças, que foram maiores especialmente na fase de estabilização da perda de massa, após a fase de degradação, ou seja, acima de $400^{\circ} \mathrm{C}$. Essa análise sugere que, embora em altas temperaturas (acima de $400^{\circ} \mathrm{C}$ ) os diferentes tipos de serragem apresentem diferenças de perdas de massa, na temperatura de processamento dos compósitos (abaixo de $200^{\circ} \mathrm{C}$ ), as perdas de massa serão pequenas e semelhantes. Essa perda, conforme o gráfico, se deve situar entre 4 e $8 \%$ e se atribui aos materiais voláteis dispersos nas fibras de madeira, os quais incluem a água.

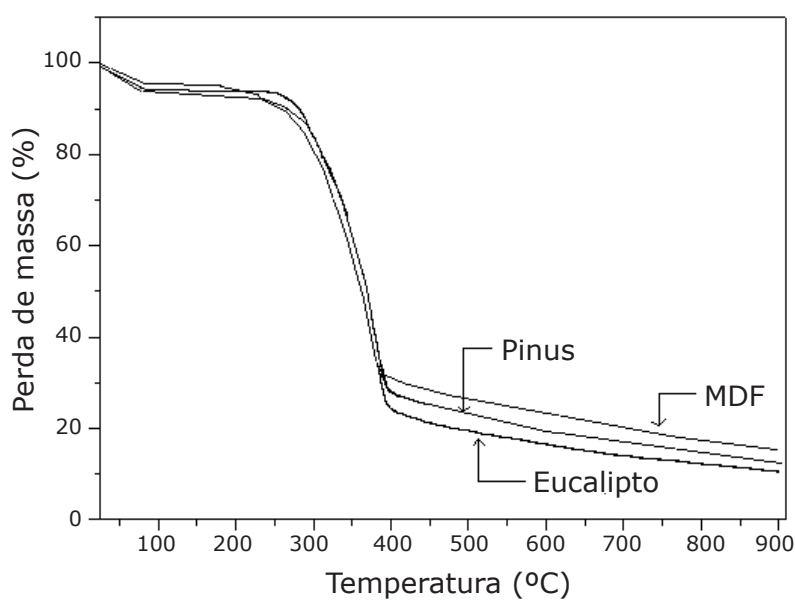

FIGURA 2: TGA das amostras dos diferentes tipos de serragem utilizados.

FIGURE 2: TGA of samples of the different sawdust types used.
Cristalinidade dos compósitos confeccionados com $30 \%$ de serragem

$\mathrm{Na}$ Tabela 4, são apresentados os índices de cristalinidade, juntamente com os valores de entalpia e de temperatura de fusão, para os compósitos confeccionados com $30 \%$ de serragem.

$\mathrm{O}$ valor-padrão de $\Delta \mathrm{H}^{\circ}$, considerando o polietileno $100 \%$ cristalino, foi de $293 \mathrm{~J} / \mathrm{g}$ (SILVA et al., 2001). Para o polietileno puro foi encontrado um valor para a temperatura do pico na cristalização de $114,3^{\circ} \mathrm{C}$ e um $\Delta \mathrm{H}_{\mathrm{c}}$ de $182,6 \mathrm{~J} / \mathrm{g}$. Para o segundo aquecimento, foi encontrada uma temperatura de fusão de $132,3^{\circ} \mathrm{C}$ e uma energia $\Delta \mathrm{H}_{\mathrm{c}}$ de $180,5 \mathrm{~J} / \mathrm{g}$. $\mathrm{O}$ índice de cristalinidade foi calculado em 0,61.

Os valores das temperaturas de fusão referentes ao primeiro e segundo ciclo de aquecimento sofreram alterações, quando comparadas ao v-HDPE puro. As amostras quando foram submetidas ao segundo aquecimento reorganizaram suas estruturas e apresentaram uma variação de entalpia maior. Todos os compósitos processados apresentaram índice de cristalinidade maior que o v-HDPE puro. Observou-se que a serragem, independente da espécie de madeira, age como nucleante, ou seja, faz com que o polímero consiga atingir, em menor tempo, uma maior proporção de material cristalino.

\section{Microscopia eletrônica de varredura}

$\mathrm{Na}$ Figura 3, são apresentadas algumas micrografias da microscopia eletrônica de varredura (SEM) da fratura de amostras dos compósitos confeccionados com extrusora de dupla-rosca. Pode-se verificar uma influência do tipo de serragem usada na dispersão das fibras na matriz polimérica.

Nas serragens deeucalipto, houve a formação de agregados de fibras, que não se dispersaram

TABELA 4: Valores de entalpia e de temperatura para a primeira fusão, segunda fusão e cristalização, dos compósitos confeccionados com $30 \%$ de serragem.

TABLE 4: Enthalpy values and temperature for the first fusion, second fusion and crystallization of composites made with $30 \%$ sawdust.

\begin{tabular}{|c|c|c|c|c|c|}
\hline Amostras & $\Delta \mathrm{H}_{\mathrm{F} 1}(\mathrm{~J} / \mathrm{G}) \mathrm{T}_{\mathrm{pl}}\left({ }^{\circ} \mathrm{C}\right)$ & $\mathrm{X}_{\mathrm{CF} 1}$ & $\Delta \mathrm{H}_{\mathrm{C}}(\mathrm{J} / \mathrm{G}) \mathrm{T}_{\mathrm{PC}}\left({ }^{\circ} \mathrm{C}\right)$ & $\Delta \mathrm{H}_{\mathrm{F} 2}(\mathrm{~J} / \mathrm{G}) \mathrm{T}_{\mathrm{P} 2}\left({ }^{\circ} \mathrm{C}\right)$ & $\mathrm{X}_{\mathrm{CF} 2}$ \\
\hline v-HDPE puro & ---- ---- & ---- & 182,$6 ; 114,3$ & 180,$5 ; 132,3$ & 0,61 \\
\hline MDF $30 \%$ DR & 117,$7 ; 132,2$ & 0,57 & 135,$3 ; 114,7$ & 135,$4 ; 133,9$ & 0,66 \\
\hline Eucalipto $30 \%$ DR & 105,$7 ; 132,5$ & 0,51 & 148,$2 ; 114,2$ & 146,$6 ; 133,8$ & 0,71 \\
\hline Pinus $30 \% \mathrm{DR}$ & 113,$1 ; 132,3$ & 0,55 & 139,$5 ; 114,5$ & 138,$1 ; 133,8$ & 0,67 \\
\hline
\end{tabular}

NOTAS: DR = Dupla rosca; $\Delta \mathrm{H}_{\mathrm{F} 1}=$ entalpia de fusão do primeiro aquecimento; $\mathrm{T}_{\mathrm{pl}}=$ temperatura de fusão do primeiro aquecimento; $\mathrm{X}_{\mathrm{cF} 1}$ = índice de cristalinidade do primeiro aquecimento; $\Delta \mathrm{H}_{\mathrm{c}}=$ entalpia de cristalização; $\mathrm{T}_{\mathrm{pc}}=$ temperatura de cristalização; $\Delta \mathrm{H}_{\mathrm{F} 2}=$ entalpia de fusão do segundo ciclo de aquecimento; $\mathrm{T}_{\mathrm{p} 2}=$ temperatura de fusão do segundo aquecimento; $\mathrm{X}_{\mathrm{cF} 2}=$ índice de cristalinidade do segundo aquecimento. 


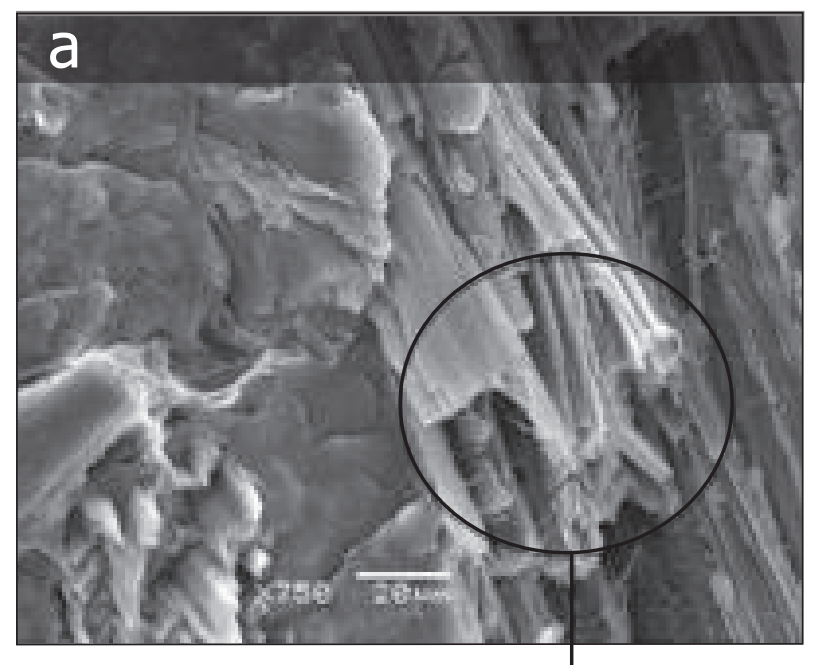

Agregado de fibras

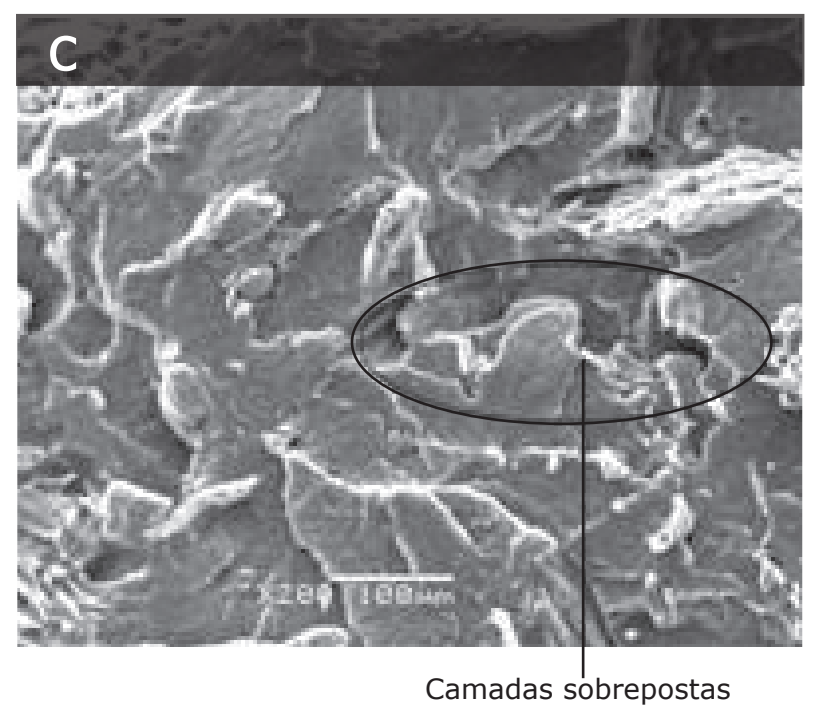

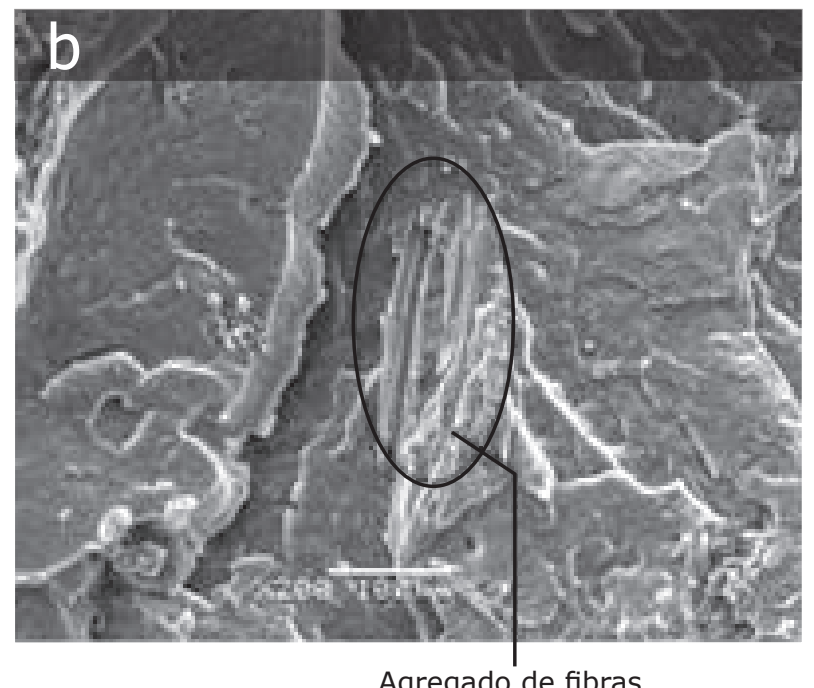

Agregado de fibras

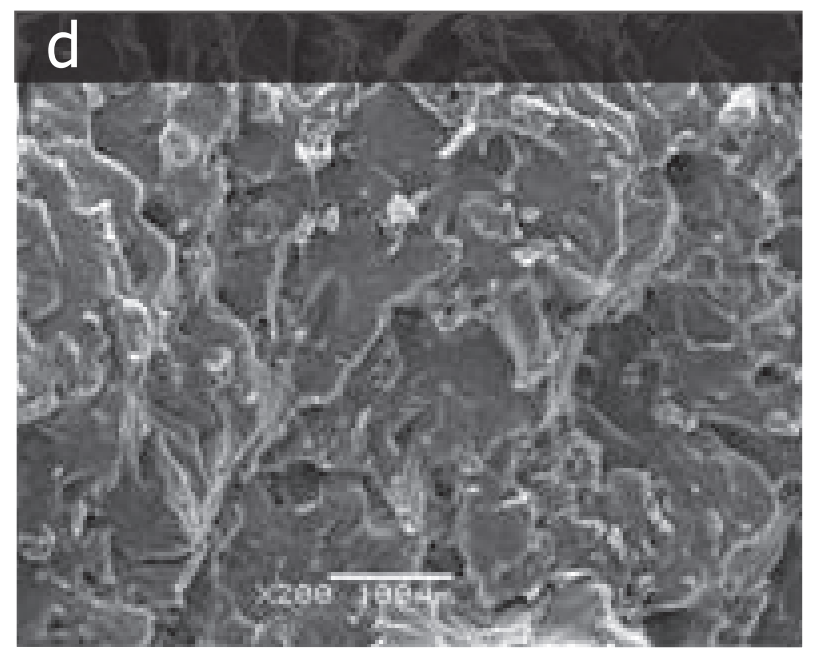

$a=$ HDPE/Eucalipto 70/30; $b=$ HDPE/Eucalipto 70/20; $c=$ HDPE/Pinus 70/30; $d=$ HDPE/MDF 70/30.

FIGURA 3: Micrografias das superfícies de fratura dos compósitos confeccionados com v-HDPE e diferentes tipos de serragem.

FIGURE 3: Micrographs of the fracture surfaces of composites made with different types of sawdust.

e que se apresentam em maior quantidade quanto maior a proporção de serragem $(a, b)$. Comparando a dispersão da serragem de eucalipto, em relação às serragens de pinus e MDF (c, d, respectivamente), verifica-se que não ocorre a formação de agregados de fibras e a matriz polimérica apresenta um aspecto homogêneo. Pode-se ver também que, para serragem de pinus, a homogeneidade da matriz é ainda maior, com menor ocorrência de camadas sobrepostas. Por outro lado, em todos os compósitos, nota-se uma boa adesão da serragem na matriz polimérica, pois não há espaços vazios entre a fibra e a matriz.

\section{Ensaios físico-mecânicos}

Na Tabela 5, são apresentados os valores médios das propriedades físico-mecânicas, para cada tipo e proporção de serragem usada nos compósitos e também para o v-HDPE puro. Os resultados mostraram que as propriedades físicomecânicas dos compósitos diferem das do v-HDPE puro, e que existem diferenças entre as propriedades dos compósitos confeccionados com diferentes tipos de serragem. Também foi observado que os valores diferem dos encontrados para os compósitos processados em extrusora mono-rosca no trabalho de Hillig et al. (2008). 
TABELA 5: Valores médios das propriedades físico-mecânicas dos compósitos confeccionados com diferentes tipos e proporções de serragem e v-HDPE

TABLE 5: Average values for the physical and mechanical properties of the composites made with different types and ratios of sawdust.

\begin{tabular}{|c|c|c|c|c|c|c|c|c|c|c|}
\hline M & Material & $\begin{array}{c}\text { Prop. de } \\
\text { serragem } \\
\quad(\%)\end{array}$ & $\begin{array}{c}\text { Massa } \\
\text { específica } \\
\left(\mathrm{g} / \mathrm{cm}^{3}\right)\end{array}$ & $\begin{array}{l}\text { Resist } \\
\text { à tração }\end{array}$ & $\begin{array}{l}\text { tência } \\
(\mathrm{MPa})\end{array}$ & $\begin{array}{l}\text { Resis } \\
\text { à flexãc }\end{array}$ & $\begin{array}{l}\text { tência } \\
\text { o (MPa) }\end{array}$ & & & $\begin{array}{c}\text { Impacto } \\
(\mathrm{J} / \mathrm{m})\end{array}$ \\
\hline 0 & v-HDPE & 0 & $\begin{array}{l}0,906 \mathrm{a} \\
(0,0085)\end{array}$ & $\begin{array}{l}19,36 \mathrm{a} \\
(2,18)\end{array}$ & & $\begin{array}{l}19,45 \mathrm{a} \\
(0,21)\end{array}$ & & $\begin{array}{l}568,0 \text { a } \\
(54,3)\end{array}$ & & $\begin{array}{l}62,01 \\
(4,88) \\
\end{array}$ \\
\hline 1 & Eucalipto & 20 & $\begin{array}{l}0,959 \quad \mathrm{c} \\
(0,0015)\end{array}$ & $\begin{array}{l}26,23 \\
(0,29)\end{array}$ & $\mathrm{c}, \mathrm{d}$ & $\begin{array}{l}32,16 \\
(0,25)\end{array}$ & $\mathrm{c}$ & $\begin{array}{l}1318,1 \\
(194,7)\end{array}$ & $\mathrm{b}, \mathrm{c}$ & $\begin{array}{ll}61,89 & d \\
(10,75) & \end{array}$ \\
\hline 2 & Eucalipto & 30 & $\begin{array}{l}0,989 \text { e } \\
(0,0023)\end{array}$ & $\begin{array}{l}28,57 \\
(0,36)\end{array}$ & $\mathrm{e}$ & $\begin{array}{l}39,61 \\
(0,65)\end{array}$ & $\mathrm{f}$ & $\begin{array}{l}1716,7 \\
(68,2)\end{array}$ & $\mathrm{c}, \mathrm{d}$ & $\begin{array}{ll}54,21 & \mathrm{c}, \mathrm{d} \\
(2,42) & \end{array}$ \\
\hline 3 & Pinus & 20 & $\begin{array}{l}0,964 \quad c \\
(0,0034)\end{array}$ & $\begin{array}{l}23,67 \mathrm{~b} \\
(1,19)\end{array}$ & & $\begin{array}{l}27,31 \mathrm{~b} \\
(0,23)\end{array}$ & & $\begin{array}{l}1104,7 \\
(97,5)\end{array}$ & $\mathrm{b}$ & $\begin{array}{l}46,71 \quad b, c \\
(2,77)\end{array}$ \\
\hline 4 & Pinus & 30 & $\begin{array}{l}0,997 \text { e } \\
(0,0030)\end{array}$ & $\begin{array}{l}24,48 \text { b, } \\
(0,06)\end{array}$ & & $\begin{array}{l}35,12 \\
(0,58) \\
\end{array}$ & d & $\begin{array}{l}1501,6 \\
(98,1) \\
\end{array}$ & $\mathrm{b}, \mathrm{c}$ & $\begin{array}{l}41,83 \text { a,b } \\
(2,07)\end{array}$ \\
\hline 5 & Pinus & 40 & $\begin{array}{ll}1,025 \quad f \\
(0,0018 & \\
\end{array}$ & $\begin{array}{l}31,08 \\
(0,25) \\
\end{array}$ & $\mathrm{f}$ & $\begin{array}{l}45,52 \\
(1,77) \\
\end{array}$ & $\mathrm{h}$ & $\begin{array}{l}1996,5 \\
(331,1) \\
\end{array}$ & $d$ & $\begin{array}{l}40,88 \mathrm{a}, \mathrm{b} \\
(1,42)\end{array}$ \\
\hline 6 & MDF & 20 & $\begin{array}{l}0,950 \mathrm{~b} \\
(0,0026)\end{array}$ & $\begin{array}{l}28,35 \\
(0,48) \\
\end{array}$ & $\mathrm{d}, \mathrm{e}$ & $\begin{array}{l}36,61 \\
(0,26) \\
\end{array}$ & $\mathrm{e}$ & $\begin{array}{l}1509,4 \\
(24,0) \\
\end{array}$ & $\mathrm{b}, \mathrm{c}$ & $\begin{array}{l}37,77 \mathrm{a} \\
(1,69)\end{array}$ \\
\hline 7 & MDF & 30 & $\begin{array}{l}0,979 \quad d \\
(0,0057)\end{array}$ & $\begin{array}{l}32,20 \\
(0,81)\end{array}$ & $f$ & $\begin{array}{l}42,69 \\
(0,25)\end{array}$ & $\mathrm{g}$ & $\begin{array}{l}1257,2 \\
(441,8)\end{array}$ & $\mathrm{b}$ & $\begin{array}{l}39,62 \text { a,b } \\
(1,13)\end{array}$ \\
\hline 8 & MDF & 40 & $\begin{array}{l}1,022 \quad f \\
(0,0016)\end{array}$ & $\begin{array}{l}35,14 \\
(1,65)\end{array}$ & & $\begin{array}{l}49,48 \\
(0,63)\end{array}$ & & $\begin{array}{l}2154,1 \\
(240,2)\end{array}$ & d & $\begin{array}{l}38,80 \mathrm{a}, \mathrm{b} \\
(0,11)\end{array}$ \\
\hline
\end{tabular}

NOTAS: Valores médios de cinco corpos-de-prova injetados. Valores entre parênteses referem-se ao Desvio-Padrão. Médias de mesmas letras não diferem pelo teste de Tukey ao nível de 5\% de significância.

\section{Massa específica}

Os valores médios de massa específica aparente dos compósitos variaram de 0,906 g/ $\mathrm{cm}^{3}$ para o polímero puro até $1,025 \mathrm{~g} / \mathrm{cm}^{3}$ para o compósito com $40 \%$ de serragem de pinus. Verificou-se que a serragem causa um aumento da massa específica dos compósitos em relação ao polímero puro e que um aumento da proporção de serragem no compósito causa um aumento da massa específica. Foram verificadas também diferenças significativas entre compósitos confeccionados com mesma proporção, mas com diferentes tipos de serragem. Além disso, foi verificado que os corpos-de-prova confeccionados com HDPE puro apresentaram massa específica um pouco maior que o valor informado na Tabela 1 (Valor de massa específica do HDPE antes do processamento, igual $\left.0,959 \mathrm{~g} / \mathrm{cm}^{3}\right)$. A influência da proporção de serragem na massa específica dos compósitos foi também verificada em outros trabalhos (Youngquist, 1999; Stark, 1999; Yam et al., 1990; Fonseca, 2005).

Pode-se inferir que, tanto no processo de extrusão como na injeção, o HDPE e as partículas de madeira sofrem compressão que tende a eliminar a porosidade da madeira. Sabe-se que a massa específica da parede celular varia de 1,50 a 1,56 g/ $\mathrm{cm}^{3}$, independente da espécie. Dessa forma, o fato de haver diferenças entre compósitos de mesma proporção, mas de diferentes tipos de serragem, mostra que a massa específica do compósito depende da estrutura anatômica da madeira, ou seja, do tipo e da distribuição dos meatos e lumens celulares (porosidade). Por outro lado, como o HDPE não é poroso, a massa específica do compósito é fortemente dependente da proporção de serragem. Conclui-se que a massa específica do compósito 
é resultado da interação dos fatores proporção de serragem e estrutura anatômica (tipo de serragem).

\section{Resistência à tração}

A resistência à tração pode explicitar como ocorre a adesão entre as fases de fibra e matriz. Num sistema ideal, se desejaria obter uma força de adesão entre as fases maior que a força de coesão entre as moléculas da fase que possui melhor propriedade. A resistência à tração para madeira, contudo, depende de vários fatores inerentes às características de um material anisotrópico. Além disso, no caso da serragem, não se refere à resistência das fibras individuais da madeira, mas, sim, da resistência de um agregado, formado por fibras e por outros tipos celulares.

Observando a Tabela 5, verifica-se que o tipo de serragem que apresentou os melhores resultados foi o MDF, na proporção de $40 \%$. Para essa mistura o valor médio de resistência à tração foi de 35,14 MPa. O valor médio mais baixo foi da ordem de 23,67 MPa, obtido para os compósitos produzidos com pinus na proporção de $20 \%$. Esse valor foi superior ao valor da resistência à tração do v-HDPE, de 19,36 MPa, apresentando diferença estatisticamente significativa deste. Dessa forma, todos os compósitos produzidos apresentaram valores de resistência à tração superior ao v-HDPE puro, confirmando que todos os tipos de serragem apresentaram boa adesão com a matriz.

Numa análise da resistência à tração em função da proporção de serragem, verifica-se que ela aumenta com o aumento da proporção de serragem. Os estudos que consideraram os efeitos da proporção de serragem na resistência à tração de compósitos de madeira com matrizes termoplásticas, apresentam resultados controversos. Robin e Breton (2001) não encontraram uma relação entre a proporção de serragem de Picea sp. e a resistência à tração de compósitos com HDPE, a qual se verificou com valor máximo a $10 \%$ de proporção de serragem. Acima desse percentual, a resistência à tração diminuiu com o aumento da proporção de serragem.

Yamaji (2004) observou que o aumento da proporção de madeira, de 10 para $30 \%$, em compósitos com PP, provocou uma diminuição da resistência à tração, tanto para compósitos confeccionados com serragem como para aqueles confeccionados com pó-de-lixa. Assim, presume-se que uma relação diretamente proporcional entre a proporção de serragem e a resistência à tração só irá ocorrer se houver uma boa dispersão da serragem na matriz polimérica e se houver uma boa adesão entre as fases, ou seja, se a força de adesão for superior a força de coesão da matriz.

Como para todos os tipos de serragem foi encontrada essa relação (Figura 4), pode-se inferir que houve melhor dispersão e melhor adesão entre as fases para a serragem de MDF e de pinus, em relação à de eucalipto. $\mathrm{O}$ fato dos compósitos de serragem de pinus ter valores médios de resistência à tração inferiores aos demais deve estar relacionado ao fato da madeira dessa espécie apresentar propriedades mecânicas inferiores. Para eucalipto, o fato de um aumento da proporção de madeira provocar menor aumento proporcional do que para pinus e MDF, indica que a adesão foi inferior. Esses fatores confirmam que o processamento em extrusora dupla-rosca proporciona uma boa dispersão na matriz polimérica.

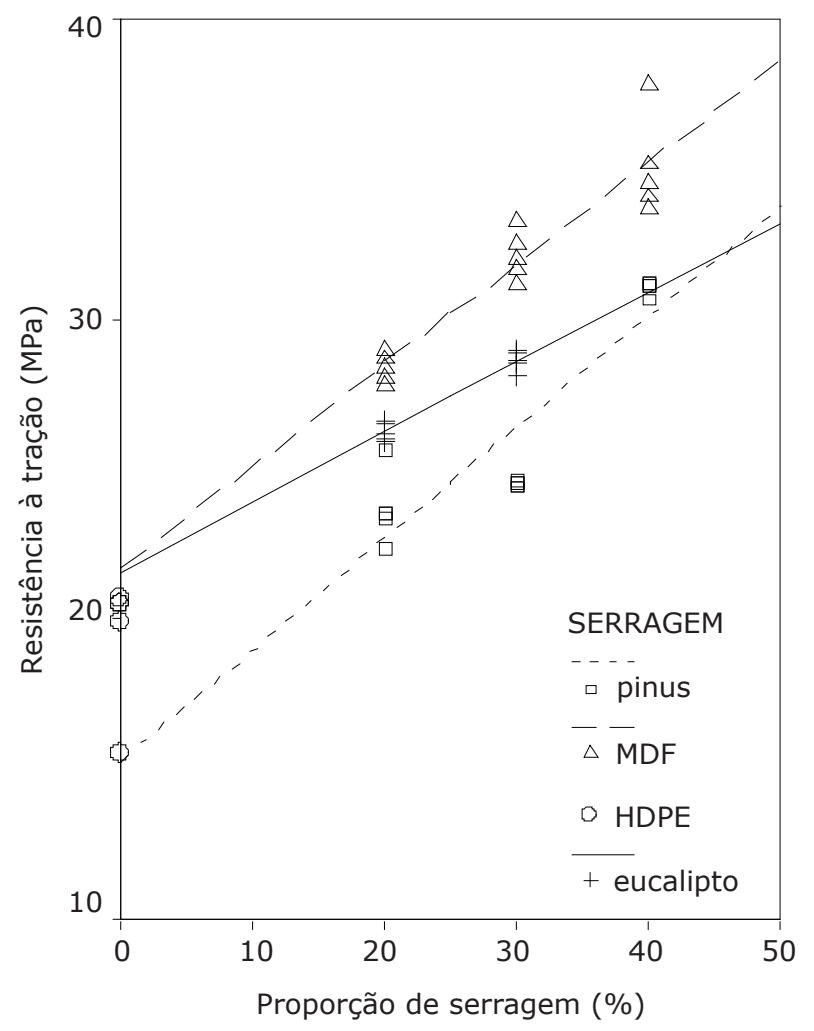

*Linhas de tendência confirmadas pela análise de variância.

FIGURA 4: Resistência à tração dos compósitos confeccionados com v-HDPE em função do tipo e proporção de serragem usada.

FIGURE 4: Tensile strength as a function of the proportion of sawdust for each type of sawdust used. 


\section{Resistência à flexão e MOE}

A análise dos dados apresentados na Tabela 5 mostra que um aumento da proporção de serragem causa um aumento da resistência à flexão. Por outro lado, se comparados aos valores apresentados em Hillig et al. (2008) para os compósitos confeccionados com mono-rosca, verifica-se que os confeccionados em duplarosca apresentaram valores de resistência à flexão ligeiramente superiores. Além disso, uma análise mais atenta mostra que esse processo tem melhor efeito na resistência à flexão do que aquele, quando se adiciona maior proporção de serragem no compósito.

A Figura 5 apresenta os valores de resistência à flexão em função da proporção de serragem, para cada tipo de serragem usada. Verifica-se que a interação entre tipo de serragem e proporção de serragem é pequena e que a serragem

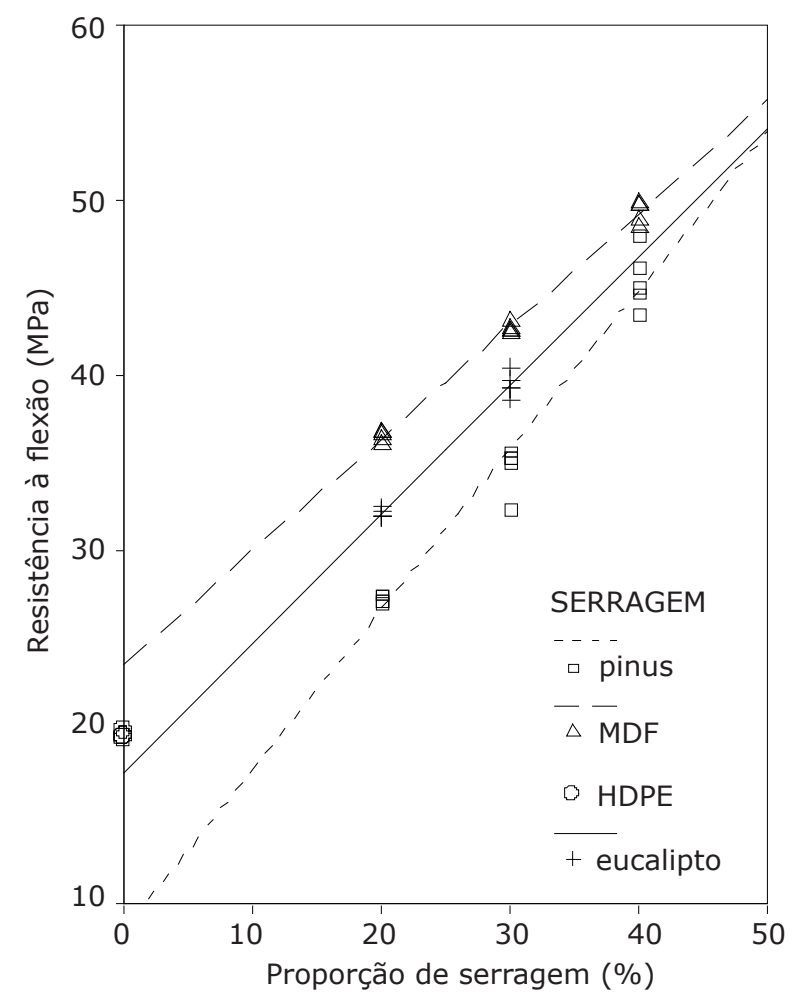

*Linhas de tendência confirmadas pela análise de variância.

FIGURA 5: Resistência à flexão dos compósitos confeccionados com v-HDPE em função do tipo e proporção de serragem usada.

FIGURE 5: Static Bending as a function of the proportion of sawdust for each type of sawdust used. de pinus apresenta os menores valores de resistência à flexão para as diferentes proporções testadas, seguido do eucalipto e MDF respectivamente.

A rigidez de compósitos de termoplásticos com madeira pode ser determinada por meio do MOE à flexão. Sain et al (2005), trabalhando com fibras de Hemp (Cannabis sp.) e fibras obtidas por processo Kraft em compósitos com PP, verificaram que os compósitos com fibra de hemp apresentaram maior MOE à flexão que os compósitos com fibras de processo Kraft.

Em ambos os casos, no entanto, os valores médios foram inferiores ao MOE obtido com compósitos de PP e fibras de vidro. Wolcott (2003), trabalhando com wood plastic composites (WPC) com base em resíduos de madeira de Loblolly pine e Douglas fir, verificou que o MOE dos compósitos com Loblolly pine foi significativamente maior que os de Douglas fir. O autor salienta que apesar do MOE e da massa específica de ambas as madeiras serradas dessas espécies serem idênticos, o MOE dos compósitos de madeira de Loblolly pine foi $78 \%$ maior que o MOE dos compósitos de Douglas fir. Essa diferença foi atribuída à diferente interação entre madeira e plástico nos compósitos. Dessa forma, os resultados sugerem que a espécie de madeira deve ter uma influência significativa na estrutura do material e, em consequência, nas suas propriedades.

Neste trabalho, observando a Tabela 5, verifica-se que não houve diferença de MOE entre os três tipos de serragem usadas, fato esse confirmado quando se aplica uma Análise de Variância para o fator tipo de serragem. No entanto, foi verificada diferença entre as proporções de serragem, sendo que um aumento da proporção de serragem causa um aumento do MOE. O aumento do MOE com o aumento da proporção de serragem, ou de fibras, em compósitos de poliolefinas com madeira foi verificado por diversos autores como Robin e Breton (2001), Stark e Rowlands (2003), Caraschi e Leão (2002), Stark (1999), entre outros.

\section{Resistência ao Impacto}

A incorporação de serragem causa uma diminuição da resistência ao impacto em relação ao HDPE puro e um aumento da sua proporção no compósito diminui ainda mais essa resistência, em linhas gerais (Tabela 4). Alguns autores verificaram que o aumento da proporção de serragem no compósito causa a diminuição de sua resistência ao impacto. Robin e Breton (2001) associaram esse

Ci. Fl., v. 21, n. 2, abr.-jun., 2011 
comportamento com o menor alongamento à flexão encontrado para os compósitos confeccionados com maior proporção de madeira.

Em outros trabalhos como de Selke e Wichman (2004) e Balasuriya et al. (2001), foram encontrados resultados diversos quando se analisa a resistência ao impacto dos compósitos em função da proporção de serragem. Esses últimos verificaram que a resistência ao impacto de compósitos extrusados com HDPE de baixo índice de fluidez (LMFI) diminuiu com o aumento da proporção de serragem. Por outro lado, a resistência ao impacto de compósitos extrusados com HDPE de médio índice de fluidez (MMFI) aumentou até um máximo de $40 \%$ de madeira no compósito, quando decresceu rapidamente com um maior incremento da proporção de madeira.

Com relação ao tipo de serragem, verificou-se que os compósitos apresentaram diferente comportamento de resistência ao impacto. A serragem de MDF apresentou os menores valores médios, seguida do pinus e eucalipto respectivamente. Na análise por MEV (Figura 3), foi observado que os compósitos confeccionados com pinus e MDF apresentaram melhor distribuição das fibras de madeira no HDPE, enquanto os compósitos confeccionados com eucalipto apresentaram agregados de fibras em determinadas regiões.

Outro fator de influência na resistência ao impacto, embora de menor proporção, é a cristalinidade do compósito. Também nesse caso, verifica-se que não houve um comportamento natural do material, pois os compósitos que apresentaram maior índice de cristalinidade foram os confeccionados com eucalipto, que também apresentaram maiores valores de resistência ao impacto.

Dessa forma, pressupõe-se que a distribuição das partículas deve ter contribuído mais para a resistência ao impacto do que a adesão na interface e a cristalinidade do compósito. De fato, tendo as partículas de eucalipto uma granulometria média de maior tamanho, e estando distribuídas em determinadas regiões do v-HDPE, ou seja, formando agregados, proporcionariam maiores espaços entre os agregados de madeira preenchidos com v-HDPE. Como o ensaio de resistência ao impacto, segundo a norma ASTM D 256, determina essa resistência numa região relativamente pequena do corpo-de-prova, se a região não for um agregado de fibras irá proporcionar grande absorção de energia, fazendo com que os compósitos apresentem maior resistência ao impacto nas condições do ensaio.

\section{CONCLUSÕES}

Todos os tipos de serragem agiram como agentes nucleantes, pois os compósitos apresentaram índices de cristalinidade maiores que o v-HDPE puro. Houve influência da granulometria da serragem usada na sua dispersão na matriz polimérica, sendo que nos compósitos com eucalipto (maior granulometria) aparecem agregados, que ocorrem em menor proporção nos compósitos com pinus (granulometria média) e estão ausentes nos com MDF (menor granulometria).

Os ensaios mecânicos mostraram diferenças nas propriedades dos compósitos confeccionados com diferentes tipos de serragem que, em parte foram atribuídas às diferentes granulometrias de serragem usadas, mas sugerem influência de outras características inerentes à espécie de madeira. De maneira geral, serragem de MDF proporcionou compósitos com maior resistência à flexão e à tração que os compósitos confeccionados com pinus e eucalipto. No MOE, não foram encontradas diferenças significativas entre as serragens usadas e para resistência ao impacto os maiores valores foram encontrados para eucalipto, que foram semelhantes aos valores do v-HDPE puro. Verificou-se que a resistência à flexão, a resistência à tração e o MOE aumentaram com o aumento da proporção de serragem para todos os compósitos, enquanto que para resistência ao impacto ocorreu o inverso.

Comparando os valores obtidos nesse trabalho com os obtidos na extrusão com monorosca, verificou-se maiores valores de resistência à tração e à flexão com dupla-rosca e para as maiores proporções de serragem. Conclui-se que foi possível transferir as propriedades da madeira para o compósito de melhor forma na extrusão com dupla-rosca.

\section{AGRADECIMENTOS}

Os autores agradecem ao Conselho Nacional de desenvolvimento Científico e Tecnológico CNPq, à Universidade de Caxias do Sul - UCS e à Universidade Federal do Paraná - UFPR, pelo auxílio financeiro e pelos recursos humanos.

Ao professor Leonardo Canto, pelo auxílio na extrusão dos compósitos. 


\section{REFERÊNCIAS BIBLIOGRÁFICAS}

AMERICAN SOCIETY FOR TESTING AND MATERIALS. Standard test methods for detemining the Izod Pendulum Impact resistance of plastics, D 256 - 97. West Conshohocken, PA, 2000.

Standard test method for Tensile properties of plastics, D 638 - 99. West Conshohocken, PA, 2000.

Standard test method for Flexural properties of unreinforced and reinforced plastics and electrical insulating materials, D 790 - 99. West Conshohocken, PA, 2000.

BALASURIYA, P. W.; MAI, Y. -W. Mechanical properties of wood flake-polyethylene composites. Part I: effects of processing methods and matrix melt flow behaviour. Composites Part A: Applied Science and Manufacturing, v 32, p. 619-629, 2001.

BLEDZKI, A. K. et al., A comparison of compounding processes and wood type for wood fibre - PP composites. Composites Part A: Applied Science and Manufacturing, v. 36, p. 789-797, 2005.

BILLMEYER JR., F. W. Textbook of polymer science. $2^{\text {nd }}$ ed. USA: John Willey \& Sons, 1971. $598 \mathrm{p}$.

BRASKEM. Folha de dados do Polietileno de Alta Densidade HC7260LS-L. Disponível em http:// www.braskem.com.br/upload/portal_braskem/pt/ produtos_e_servicos/folha_dados/HC7260LSL_ por.pdf. Acesso em: 8 de setembro de 2010.

CARASCHI, J. C.; LEÃO, A. L. Avaliação das propriedades mecânicas dos plásticos reciclados provenientes de resíduos sólidos urbanos. Acta Scientiarum, v. 24, n. 6, p. 1599-1602, 2002.

CHEMTURA CORPORATION. Tecnical information Polybond ${ }^{\circledR}$ 3009. Disponível em http://www.chemtura.com/deployedfiles/ staticfiles/businessunits/polymer additives-en-us/ TechnicalDataSheets/files/Polybond\%203009\%20 TDS.pdf/Polybond\%203009\%20TDS.pdf. Acesso em: 8 de setembro de 2010 .

CLEMONS C. M.; IBACH, R. E. Efects of processing method and moisture history laboratory fungal resistance of wood-HDPE composites. Forest Products Journal. v. 54, n. 4, p. 50-57, 2004.

FONSECA, F. M. C. Desenvolvimento e caracterização de compósitos à base de Polietileno de Alta Densidade (PEAD) reciclado e fibras vegetais. Belo Horizonte, 2005. 133 p. Dissertação (Mestrado em Engenharia de Materiais)Universidade Federal de Ouro Preto, Ouro Preto, 2005.

HILLIG, É. et al. Caracterização de compósitos produzidos com polietileno de alta densidade (HDPE) e serragem da indústria moveleira. Revista Árvore, v. 32, n. 2, p.299-310, 2008.

ROBIN, J. J.; BRETON, Y. Reinforcement of recycled polyethylene with wood fibers heat treated. Journal of Reinforced Plastics and Composites, v. 20 , n. 14 , p. $1253-1262,2001$..

ROSE, J. Equipment overcomes - some knotty problems. Modern Plastics,March, p. 40-41, 2002. RUCH, J. et al. Transformação direta facilita a moldagem plásticos reforçados com fibras naturais. Plástico Industrial, n. 68, p. 44-63, abr. 2004.

SAHEB, D. N.; JOG, J. P. Natural fiber polymer composites: a review. Advances in Polymer Technology. v. 18, n. 4, p. 351-363, 1999.

SAIN, M. et al. Interface Modification and Mechanical Properties of Natural Fiber-Polyolefin Composite Products. Journal of Reinforced Plastics and Composites, v. 24, n. 2, p. 121-130, 2005

SELKE, S. E.; WICHMAN, I. Wood fiber polyolefin composites. Composites Part A: Applied Science and Manufacturing, v. 35, p. 321-326. 2004 STARK, N. M. Wood fiber derived from scrap pallets used in polypropylene composites. Forest Products Journal, v. 49, n. 6, p. 39-46, Jun. 1999. STARK, N. M.; MATUANA, L. M.; CLEMONS, C. M. Effect of Processing Method on Accelerated Weathering of Woodflour-HDPE Composites. In: INTERNATIONAL CONFERENCE ON WOODFIBER-PLASTIC COMPOSITES (AND OTHER NATURAL FIBERS), 7., 2003, Madison. Proceedings... Madison: Forest Products Society, 2003. p. 79-87.

STARK, N. M.; ROWLANDS R. E. Effects of wood fiber characteristics on mechanical properties of wood/polypropylene composites. Wood fiber science . 35, p. 167-174, 2003.

TECNOLOGIA PRODUZ MADEIRA PLÁSTICA. Plástico Moderno, n. 315, 2000. Disponível em http://www.plastico.com.br/revista/pm315/ extrusoras4.htm. Acesso em: 8 de setembro de 2010. VIANNA, W. L., CORREA, C. A., RAZZINO, C. A. Efeitos do tipo de poliestireno de alto impacto nas propriedades de compósitos termoplásticos com farinha de resíduo de madeira. Polímeros: Ciência 
e Tecnologia, v. 14, n. 5, p. 339-348, 2004.

WOLCOTT, M. P. Production methods and platforms for wood plastics. Non-Wood Substitutes for Solid Wood Products Conference. Melbourne, Oct. 2003.

YAM, K. L. et al. Composites from compounding wood fibers with recycled high density polyethylene. Polymer Engineering \& Science. v. 30, n. 11, p. 693-699, 1990.
YAMAJI, F. M. Produção de compósito plásticomadeira a partir de resíduos da indústria madeireira. 2004. 182 f. Tese (Doutorado em Engenharia Florestal)-Universidade Federal do Paraná, Curitiba, 2004.

YOUNGQUIST, J. A. Wood-based composites and panel products. Wood Handbook: wood as an engineering material. Washington: Forest Products Society, 1999. 428 p. 